\title{
Summary of: Atomistic Structures of Zeolite-Templated Carbon
}

Full Review Article: https://doi.org/10.1021/acs.chemmater.0c00535

\author{
Erin E. Taylor, Kaitlin Garman, and Nicholas P. Stadie \\ Department of Chemistry \& Biochemistry \\ Montana State University, Bozeman, MT 59717
}

\begin{abstract}
This document is an author created summary of a Submitted Work that was published in Chemistry of Materials, copyright 2020 ( American Chemical Society. To access the final edited and published work see https://doi.org/10.1021/acs.chemmater.0c00535
\end{abstract}

\section{Introduction}

The discovery of fullerenes in $1985^{1}$, sparked "the era of carbon allotropes" 2 , leading to the synthesis of carbon nanotubes in 19913, and the isolation of graphene in 20044. While, fullerenes, carbon nanotubes, and graphene represent zero-, one-, and two-dimensional $\mathrm{sp}^{2}$-hybridized carbon network solids, respectively, a three-dimensional carbon network solid analog has yet to be isolated in the laboratory. Such a material was first conceptualized by Jones in 19915, followed by the atomistic rendering of a triply periodic minimal surface made entirely out of $\mathrm{sp}^{2}$-hybridized carbon by MacKay and Terrones $^{6}$ later that same year. Analogous to the introduction of five-membered carbon rings in fullerenes to induce positive Gaussian curvature, the creation of negative Gaussian curvature is achieved by the introduction of rings with seven or more $\mathrm{sp}^{2}$ hybridized carbons. Schwarzites, as termed by Lenosky et al.7, are hence the missing threedimensional carbon network solids comprised entirely of $\mathrm{sp}^{2}$-hybridized carbon. Named after the triply periodic minimal surfaces discovered by German mathematician Hermann Schwarz, schwarzites are defined by zero mean curvature at every point along a surface that divides space into two identical volumes, such as the well-known Psurface, D-surface, and G-surface.

\section{Zeolite-Templated Carbon}

Zeolite-templated carbon (ZTC), first synthesized in 1992 by Kyotani et al. ${ }^{8}$, is the first experimentallyrealized example of an atomically thin threedimensional carbon network solid with an ordered porosity. Produced by a hard-casting strategy, ZTCs are synthesized via carbon deposition within an aluminosilicate zeolite template lending a desired final structural topology. In a typical synthesis, the zeolite template, most commonly faujasite (FAU), is impregnated with liquid and/or gaseous carbon precursors, and subsequently removed, leaving behind a structure composed primarily of carbon. Many zeolites exhibit topologies that are isomorphic with triply periodic minimal surfaces (e.g., FAU closely resembles a D-surface); however, it is important to note that a true triply periodic minimal surface must divide space into two identical volumes. Hence, most actual FAU templates should be identified as exhibiting a "non-balanced" triply periodic minimal surface. Any carbon network deposited within a FAU template is unlikely to result in a balanced schwarzite unless it forms a singlelayer that is offset from the template surface by the precise distance to separate space into two identical volumes.

\section{Atomistic Modeling of ZTC}

While all synthetic ZTC materials prepared to date are amorphous at the atomistic scale, pore-to-pore ordering is retained from the zeolite template, making atomistic modeling of ZTC structure a nontrivial yet conceivable task. While early atomistic modeling of ZTC was motivated by the desire to replicate experimental observations, recent models have been developed which rather consider the theoretical structure of a perfectly templated zeolite. The latter motive has recently become confusingly entangled with the former, leading to the incorrect claim that currently synthesized "ZTCs are schwarzites incarnate." 9 Herein, we compare experimental findings to all existing ZTC atomistic models and show that currently synthesized ZTCs cannot be considered schwarzites. We focus on ZTC templated within an FAU template (FAU-ZTC) as the material of interest in this review. 


\section{ZTC Characterization}

\section{$X$-ray Diffraction}

The template fidelity of ZTCs is determined using powder X-ray diffraction (XRD). Successful templating of a FAU-ZTC is indicated by the replication of the FAU (111) reflection at $2 \theta \approx 6.3^{\circ}$. Optimized procedures also result in the appearance of the FAU $(220)$ reflection at $2 \theta \approx 10.3^{\circ}$. On the other hand, poor templating is indicated by the presence of a peak at $2 \theta \approx 26^{\circ}$, corresponding to the (002) reflection of graphite. This is the result of either over-templating, i.e., graphitic growth on the outside of the zeolite template, or poor connectivity of the carbon network during synthesis, leading to collapse of the ZTC framework upon dissolution of the template.

\section{Nitrogen Adsorption}

Nitrogen $\left(\mathrm{N}_{2}\right)$ adsorption at $77 \mathrm{~K}$ is used to determine the Brunauer-Emmett-Teller (BET) surface area (SA) and non-local density functional theory (NLDFT) pore size distribution (PSD) of the resulting microporous ZTC. Archetypical laboratorysynthesized ZTCs have BET surface areas ranging from 3200-3800 $\mathrm{m}^{2} \mathrm{~g}^{-1}$, much larger than that of double-sided graphene; this is indicative of a structure that is accessible to $\mathrm{N}_{2}$ on both sides and additionally possesses a significant quantity of edge sites. Well-replicated ZTCs show a narrow PSD centered around $1.2 \mathrm{~nm}$, indicating atomically thin connectivity of the carbon structure (having a poreto-pore repeat distance of only $1.4 \mathrm{~nm}$ ).

\section{Thermogravimetric Analysis}

Thermogravimetric analysis (TGA) is used to determine the amount of carbonaceous material deposited within the zeolite template. Upon heating the zeolite-ZTC composite to $800{ }^{\circ} \mathrm{C}$ in air, the carbonaceous material can be completely oxidized leaving behind only the original zeolite. By analyzing the initial and final mass, the experimental structural packing density $\left(\mathrm{SPD}_{\exp }\right)$ can be determined, as defined below:

$$
\mathrm{SPD}_{\exp }=\frac{\mathrm{m}_{\mathrm{i}}-\mathrm{m}_{\mathrm{f}}}{\mathrm{m}_{\mathrm{f}}}=\frac{\mathrm{m}_{\text {carbon }}}{\mathrm{m}_{\text {zeolite }}}
$$

In Equation 1, $\mathrm{m}_{\mathrm{i}}$ is the mass of the dried zeolite-ZTC composite and $\mathrm{m}_{\mathrm{f}}$ is the mass of the untemplated zeolite. This metric is useful when comparing ZTCs synthesized on exactly the same zeolite template (e.g., a faujasite-type zeolite with a Si:Al ratio of 5.5 charge balanced with sodium cations). However, even zeolites of the same type may contain different Si:Al ratios or different charge balancing cations. Thus, SPD $_{\text {exp }}$ cannot be used to compare ZTCs synthesized within different zeolite templates. We propose two new metrics in this work that can be used to compare ZTCs templated on slightly different templates. The first, $\mathrm{SPD}_{\text {cell }}$, can be used to compare across the same zeolite template type (e.g., FAU) and the second, $\mathrm{SPD}_{\text {cell,vol }}$, can be used across arbitrarily different zeolites (e.g., *BEA-ZTC vs. FAU-ZTC). These metrics are defined as follows:

$$
\begin{aligned}
& \mathrm{SPD}_{\text {cell }}=\mathrm{SPD}_{\text {exp }} \times \frac{\mathrm{MW}_{\text {cell,zeolite }}}{\mathrm{MW}_{\text {cell,Sio }}} \\
& \mathrm{SPD}_{\text {cell,vol }}=\mathrm{SPD}_{\text {cell }} \times \frac{1}{\mathrm{v}_{\text {cell,pore }}}
\end{aligned}
$$

In Equations 2-3, $\mathrm{MW}_{\text {cell,zeolite }}$ is the weight of the zeolite's unit cell, $\mathrm{MW}_{\mathrm{cell}, \mathrm{SiO}_{2}}$ is the weight of the same zeolite unit cell assuming it is comprised purely of $\mathrm{SiO}_{2}$, and $\mathrm{v}_{\text {cell,pore }}$ is the accessible pore volume of an idealized $\mathrm{SiO}_{2}$ framework (based on a $2.8 \AA$ A probe molecule as designated by the International Zeolite Association ${ }^{10}$ ). Typical SPD cell values for archetypical FAU-ZTC range from 0.35$0.40 \mathrm{~g}_{\text {carbon }} \mathrm{g}_{\mathrm{SiO}_{2}}^{-1}$. Higher $\mathrm{SPD}_{\text {cell }}$ values have been measured, but only for over-templated ZTCs where a core-shell structure is clearly observed. ${ }^{11,12}$ We do not consider such core-shell materials to be evidence for successful improvement of the carbon packing density in bulk, free-standing ZTC as a homogeneous carbon-based framework solid.

\section{ZTC Atomistic Models}

\section{Roussel Model}

The first atomistic model of FAU-ZTC was reported in 2006, with the objective of corroborating experimental $\mathrm{N}_{2}$ adsorption isotherms. ${ }^{13}$ Using grand canonical Monte Carlo (GCMC) simulations, this effort resulted in a closed-tube structure, as shown in Figure 1a. 


\section{Nishihara Model I}

In 2009, Nishihara et al. reported several atomistic models of ZTC in order to reconcile the low structural packing density experimentally observed in FAUZTC. ${ }^{14}$ Allowing the structure to contain five-, six-, and seven-membered rings, Nishihara Model 0a and Nishihara Model 0b, shown in Figure 1b and Figure 1c, were developed. In all cases explored, by forcing the deposited carbon to connect through all four entrances of the FAU supercage, the resulting SPD $_{\text {cell }}$ was much higher than seen experimentally: 0.77 and 0.45 , respectively. Thus, a new model was created, Nishihara Model I (Figure 1d), allowing the carbon to connect through only three out of the four entrances in each zeolite supercage. No seven-membered rings were present in the final structure. Hydrogen and a variety of oxygen-based functional groups (consistent with experiment) were also added after structural optimization to create the first compositionally-correct ZTC model.

\section{Nueangnoraj Model}

A new type of chemical vapor deposition (CVD) procedure, termed pulsed chemical vapor deposition (P-CVD), was reported by Nueangnoraj et al. to achieve a core-shell ZTC structure with a core SPD ${ }_{\text {cell }}$ higher than archetypical FAU-ZTC. ${ }^{11}$ A graphitic shell surrounded each core, a sign of over- deposition. Nevertheless, a closed-strut structure was suggested to be consistent with the core structure of this unique ZTC, as shown in Figure 1e. While an oxygenfunctionalized subunit of this model was also reported, the extended model is comprised solely of carbon. Unfortunately, the Nueangnoraj Model could not be characterized further in this review, as a periodic variant was not produced.

Table 1. Chemical and structural characteristics of FAU-ZTC models compared to archetypical FAU-ZTC.

\begin{tabular}{|lcccc|}
\hline Model/Material & Structure Type & $\begin{array}{c}\text { Surface Area } \\
\left(\mathrm{m}^{2} \mathrm{~g}^{-1}\right.\end{array}$ & $\begin{array}{c}\text { SPD cell } \\
\left(\mathrm{g}_{\text {carbon }} \mathrm{g}_{\mathrm{SiO}_{2}}^{-1}\right)\end{array}$ & $\begin{array}{c}\text { Chemical } \\
\text { Composition }\end{array}$ \\
\hline Roussel Model & Closed-Strut & 1850 & 0.62 & $\mathrm{C}_{100}$ \\
Nishihara Model 0a & Closed-Strut & 1350 & 0.77 & $\mathrm{C}_{100}$ \\
Nishihara Model 0b & Closed-Strut & 2630 & 0.45 & $\mathrm{C}_{100}$ \\
Nishihara Model I & Open-Blade & 4790 & 0.32 & $\mathrm{C}_{78.4} \mathrm{H}_{15.7} \mathrm{O}_{5.9}$ \\
Nueangnoraj Model & Closed-Strut & - & 0.54 & $\mathrm{C}_{100}$ \\
Kim Model & Open-Blade & 5360 & 0.21 & $\mathrm{C}_{100}$ \\
Nishihara Model II & Open-Blade & 4490 & 0.33 & $\mathrm{C}_{71.2} \mathrm{H}_{23.8} \mathrm{O}_{5.0}$ \\
Braun Model R & Closed-Strut & 1480 & 0.68 & $\mathrm{C}_{100}$ \\
Tanaka Model IV & Open-Blade & 3730 & 0.36 & $\mathrm{C}_{100}$ \\
Boonyoung Model & Closed-Strut & - & - & $\mathrm{C}_{100}$ \\
Archetypical FAU-ZTC14-17 & - & $3200-3800$ & $0.32-0.42$ & $\mathrm{C}_{75.3} \mathrm{H}_{19.0} \mathrm{O}_{5.7}$ \\
\hline
\end{tabular}

\section{Kim Model}

After templating a lanthanum-exchanged FAU template, Kim et al. utilized single crystal XRD of the zeolite-ZTC composite to obtain the Kim Model, as shown in Figure 1f. ${ }^{15}$ This model is different from previous models since it represents the measured average positions of carbon atoms in FAU-ZTC. While the structure as a whole contains too many carbon sites to be a reasonable standalone structure
$\left(\mathrm{SPD}_{\text {cell }}=1.23\right.$ when considering carbon to occupy all of the sites), assigning measured partial occupancies to each site indicates the true $S P D_{\text {cell, }}$ as reported in Table 1. Unfortunately, a reasonable standalone structure that remained connected in three-dimensions based on these measured site occupancies could not be conceived in this work, making a fair comparison of this model's properties a challenge. 
a

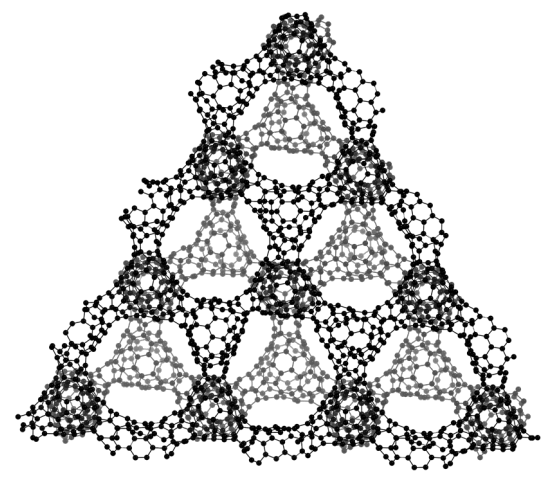

d

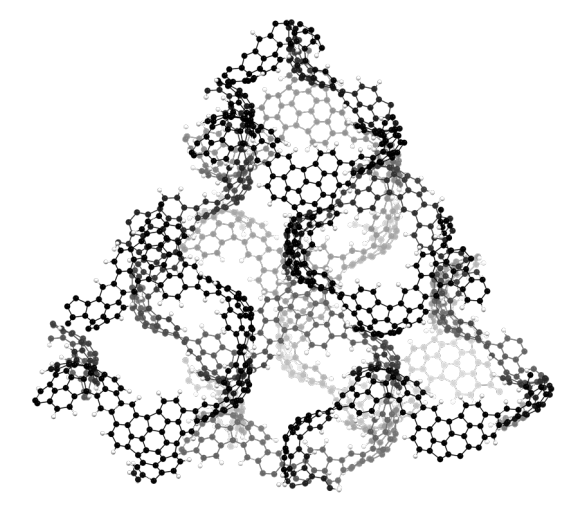

g

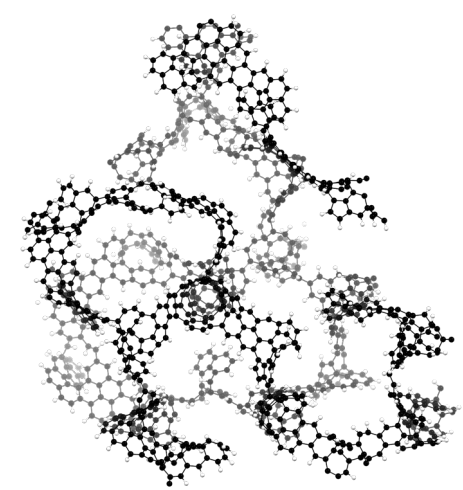

b

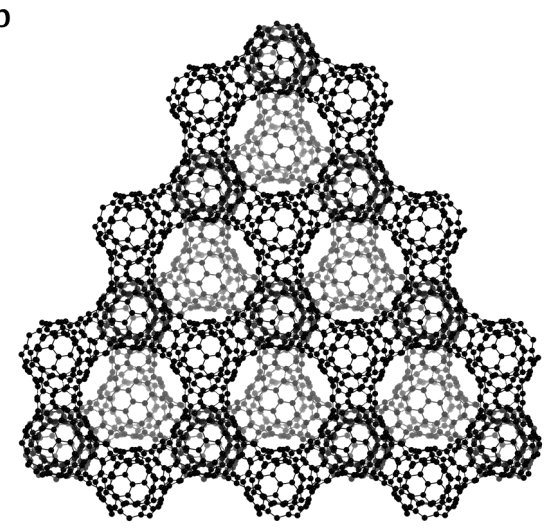

e

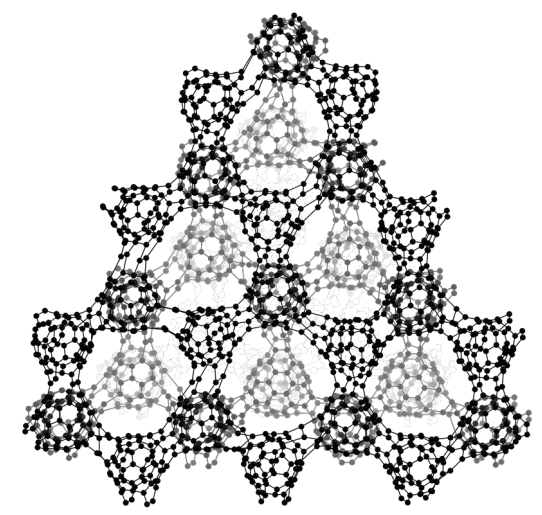

$\mathrm{h}$

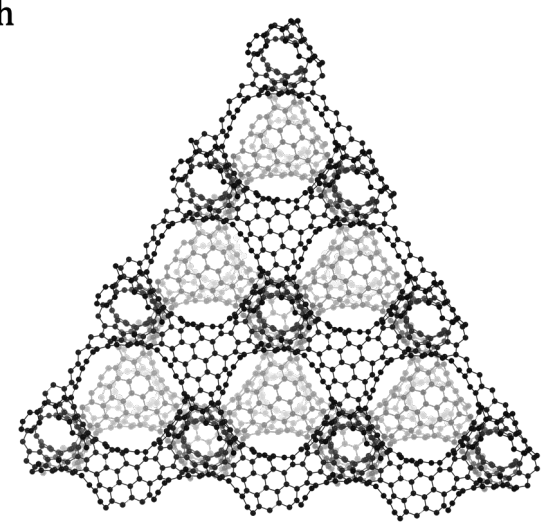

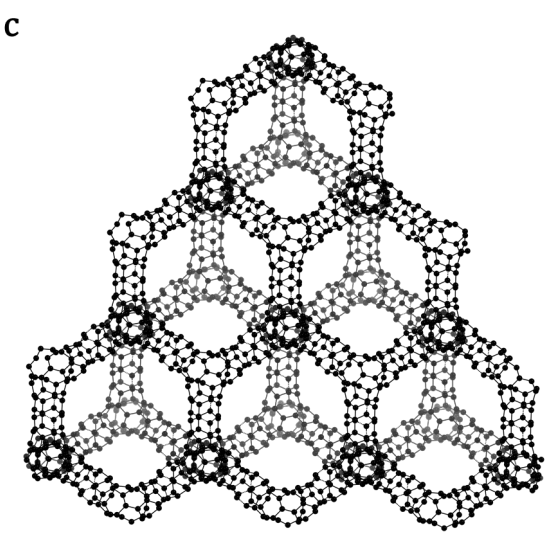

f

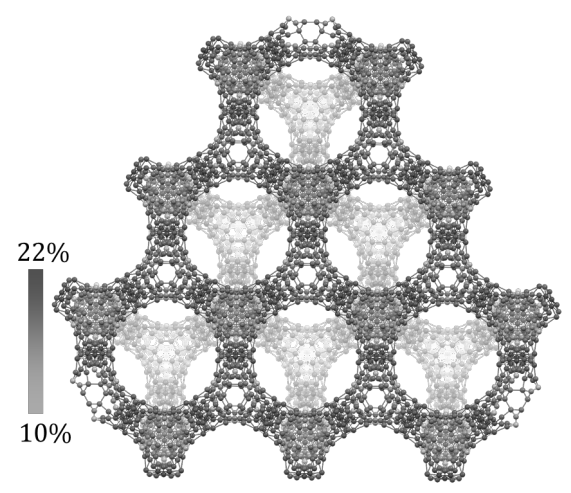

i

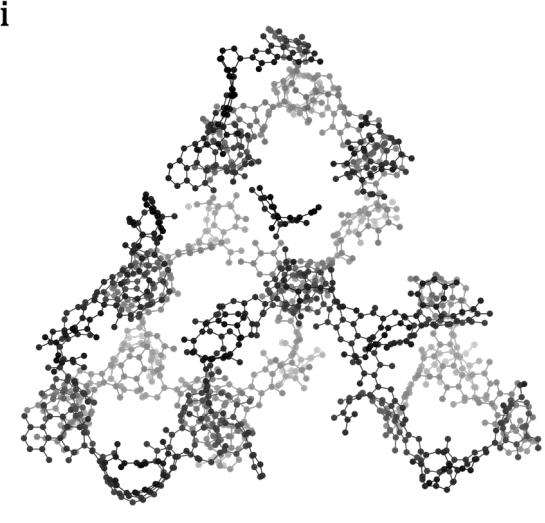

Figure 1. Representative extended multicell structures for (a) Roussel Model, (b) Nishihara Model 0a, (c) Nishihara Model 0b, (d) Nishihara Model I with hydrogen shown in white, (e) Nueangnoraj Model, (f) Kim Model with partial occupancy ranging from 22\% (dark gray) to 10\% (light gray), (g) Nishihara Model II with hydrogen shown in white, (h) Braun Model R, and (i) Tanaka Model IV. 


\section{Nishihara Model II}

In 2018, it was confirmed that ZTCs contain up to at least seven-membered rings, with possible higher ring sizes. ${ }^{18}$ To improve on past work and ensure that larger rings were represented in the atomistic model of FAU-ZTC, Nishihara et al. developed the large (comprising a $2 \times 2 \times 2$ native FAU supercell), open-blade Nishihara Model II, as shown in Figure 1g. While the original Nishihara Model II only included carbon and hydrogen, a revised model was published in $2019^{19}$ which is augmented by a similar variety of oxygen functional groups as the oxygendecorated Nishihara Model I.

\section{Braun Model}

In 2018, an extraordinary library of ZTC atomistic models was reported by Braun et al., developed using

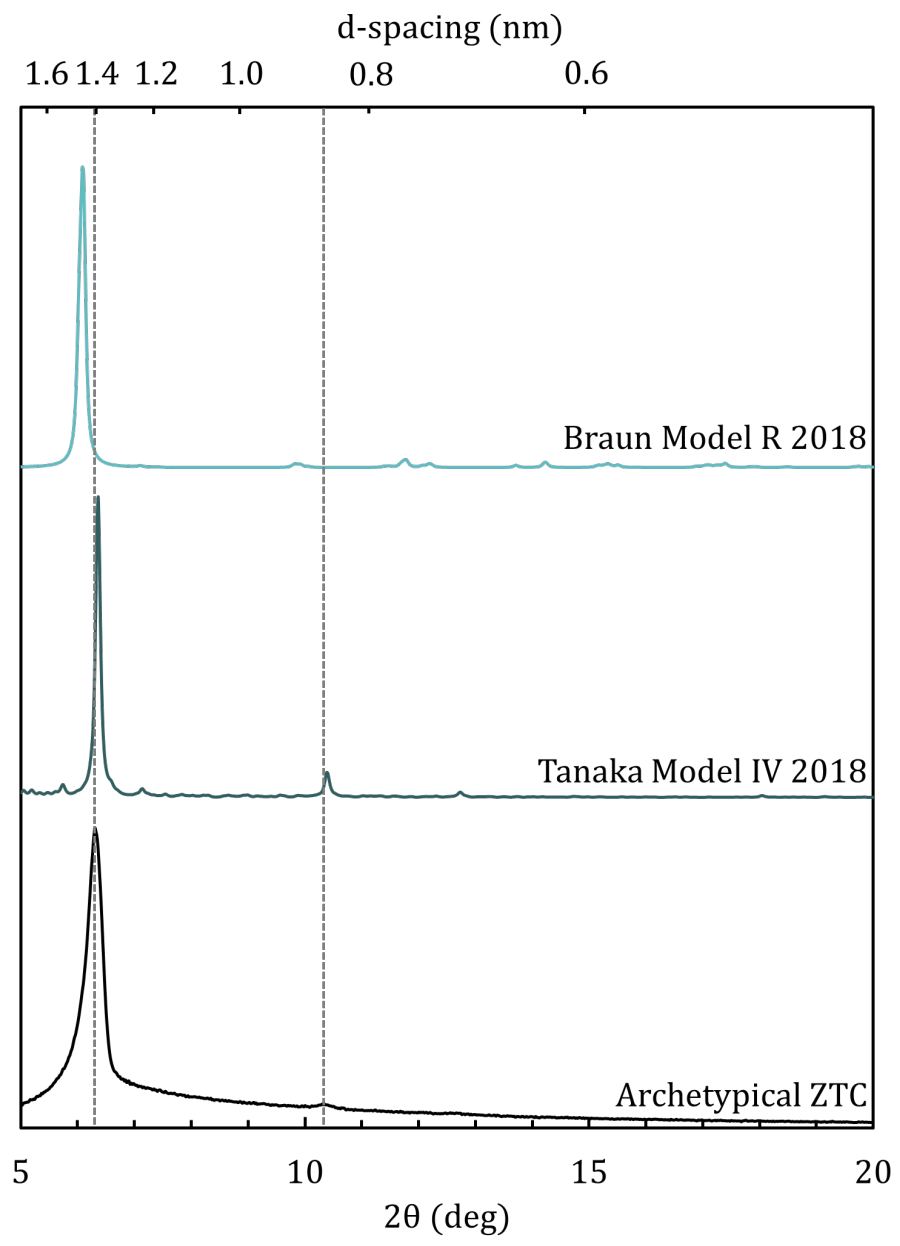

every possible zeolite template that could host a three-connected carbon structure and carried out with high-level computational methods. ${ }^{9}$ In this work, carbon atoms were positioned directly on the surface of the zeolite itself (as opposed to anywhere within the pore volume of the zeolite channels and cages). Fourteen of the proposed ZTC models, made on zeolite templates which resemble triply periodic minimal surfaces, are schwarzite-like. Two models in particular, referred to as Braun Model NR and Braun Model R (Figure 1h), correspond to FAU-ZTC structures before and after structural relaxation, respectively. Both structures are closed-strut type models with high regularity and structural packing densities $\quad\left(\mathrm{SPD}_{\text {cell }}=0.71\right.$ and $\quad \mathrm{SPD}_{\text {cell }}=0.68$, respectively).

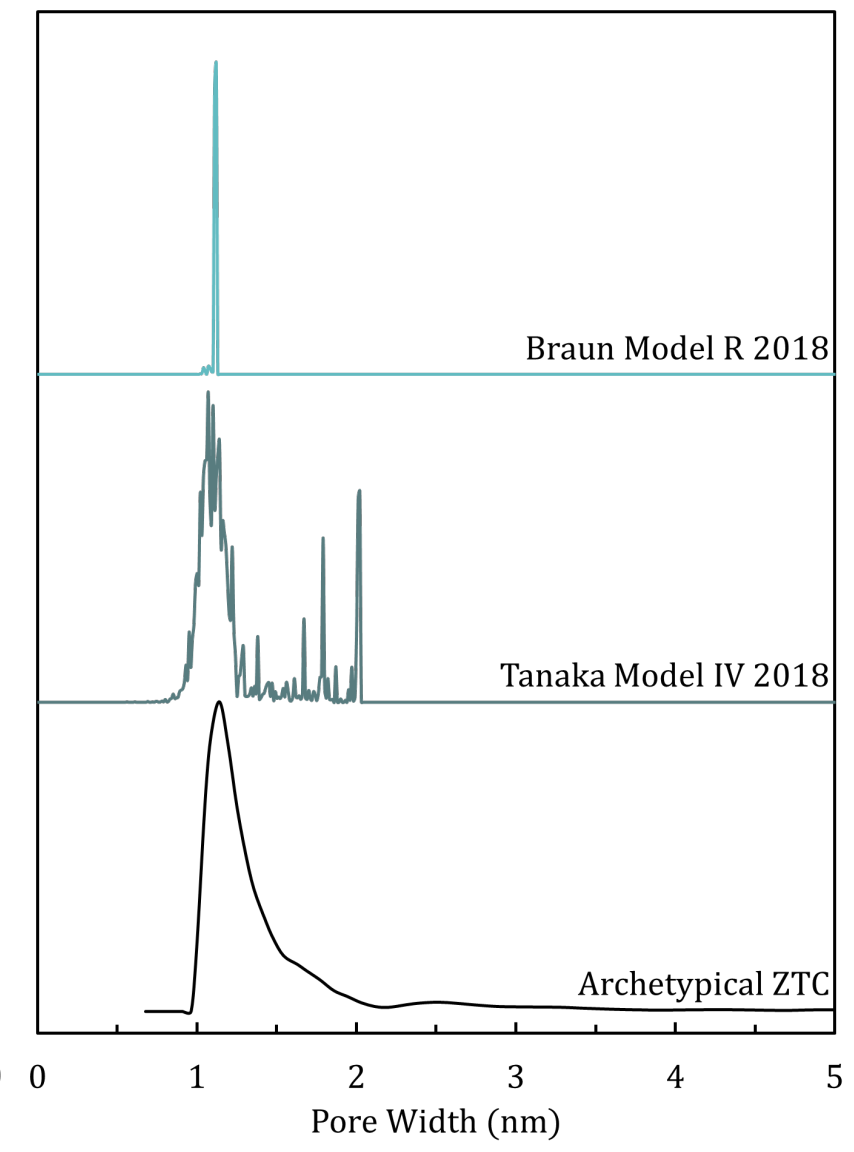

Figure 2. Experimental and theoretical X-ray diffraction patterns (left) and $\mathrm{N}_{2}$ accessible pore size distribution (right). 


\section{Tanaka Model IV}

Also in 2018, Tanaka et al. reported a library of possible ZTC structures based on a similar subset of zeolite templates. ${ }^{20}$ While Nishihara Model II is more disordered than Nishihara Model I, it was still recognized to exhibit more order and a more regular PSD than experimental ZTCs. In order to introduce more disorder into the model, carbon density was varied within a $3 \times 3 \times 3$ native $F A U$ supercell, allowing for the introduction of a few larger micropores. The result of this work is referred to herein as Tanaka Model IV, a predominantly openblade structure shown in Figure 1i.

\section{Boonyoung Model}

In an attempt to alleviate the issue of heterogeneous carbon deposition within the zeolite template during ZTC synthesis, Boonyoung et al. reported the effort to template a nanosized FAU zeolite template in 2019.12 Similar to earlier work by Nueangnoraj et al., this resulted in a core-shell ZTC structure, albeit with a higher $\mathrm{SPD}_{\text {cell }}$ in the core of each particle. A nonperiodic, closed-strut structure was developed to model the core part of these materials; unfortunately, due to the non-periodic nature of the Boonyoung Model, further characterization could not be performed in this review.

\section{Discussion}

Based on the XRD pattern (Figure 2), SA (Table 1), PSD (Figure 2), and SPD (Table 1) calculated for each of the atomistic models, it is clear that the experimental structure of FAU-ZTC is best described by a large supercell which can accommodate many different types of carbon connectivity, but comprised mainly of open-blade connectors. Without regard to chemical composition, we find Tanaka Model IV to be the most accurate representation of archetypical FAU-ZTC as currently synthesized in the laboratory. Nevertheless, incorporation of hydrogen and oxygen-based functional groups remains imperative to accomplish an even more accurate depiction of archetypical ZTC.

Recently, Braun et al. incorrectly asserted that laboratory-synthesized FAU-ZTC is a genuine example of the long-sought class of materials referred to as schwarzites. They also suggest that previous researchers in this area overlooked this equivalency and refer to their new models as an improvement over past work.

The following assertions are made in their report:

"In this work, we develop an improved molecular description of ZTCs, show that they are equivalent to schwartzites [sic], and thus make the experimental discovery of schwarzites ex post facto." (page 1$)^{9}$

"Although schwarzites have been a purely hypothetical concept, our work suggests that ZTCs are schwarzites incarnate." (page 6) ${ }^{9}$

"[W]e have shown how ZTCs can be associated with TPMSs, linking the research topics of ZTCs and schwarzites." (page 6) ${ }^{9}$

"Indeed, the experimental properties of ZTCs are exactly those which have been predicted for schwarzites..." (page 1$)^{9}$

However, as shown in this review, the experimental properties of ZTCs do not match those of schwarzites or schwarzite-like structures in three obvious ways: a mostly amorphous XRD pattern, a SA greatly exceeding two-sided graphene, and a carbon density much less than required for closed-strut connectivity between pores. In addition, laboratory-produced ZTCs contain a significant quantity of hydrogen $(\sim 2$ wt $\%)$, oxygen functionalities ( $\sim \mathrm{wt} \%)$, and a heterogeneous distribution of SPD and pore sizes throughout the structure. The chemical composition alone is disqualifying for any comparison between ZTCs and schwarzites. On the other hand, both Nishihara Model II and Tanaka Model IV represent mostly amorphous carbon networks with properties that accurately represent experimental FAU-ZTC. The Braun Models (NR and R) depict a highly symmetrical carbon network with very little disorder and heterogeneity, no edge sites, and no possibility for oxygen incorporation. A direct comparison between Tanaka Model IV and Braun Model R is shown in Figure 3.

While the conclusions put forth by Braun et al. are inaccurate, their work nevertheless represents a valuable accomplishment in compiling a collection of 
possible "non-balanced" schwarzites which could one day exist in the form of ZTCs if the templating of carbon could be carried out directly and exclusively at the zeolite surface. Our main emphasis in this review is that while the claims of Braun et al. have been repeated (wholly unsubstantiated) in recent literature ${ }^{21-24}$, schwarzites still remain elusive in the synthetic laboratory.
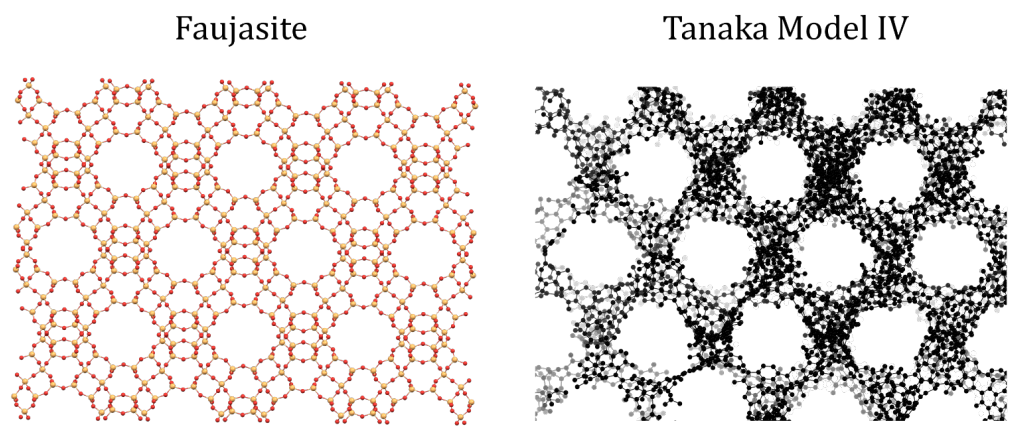

Braun Model R

$\langle 111\rangle$
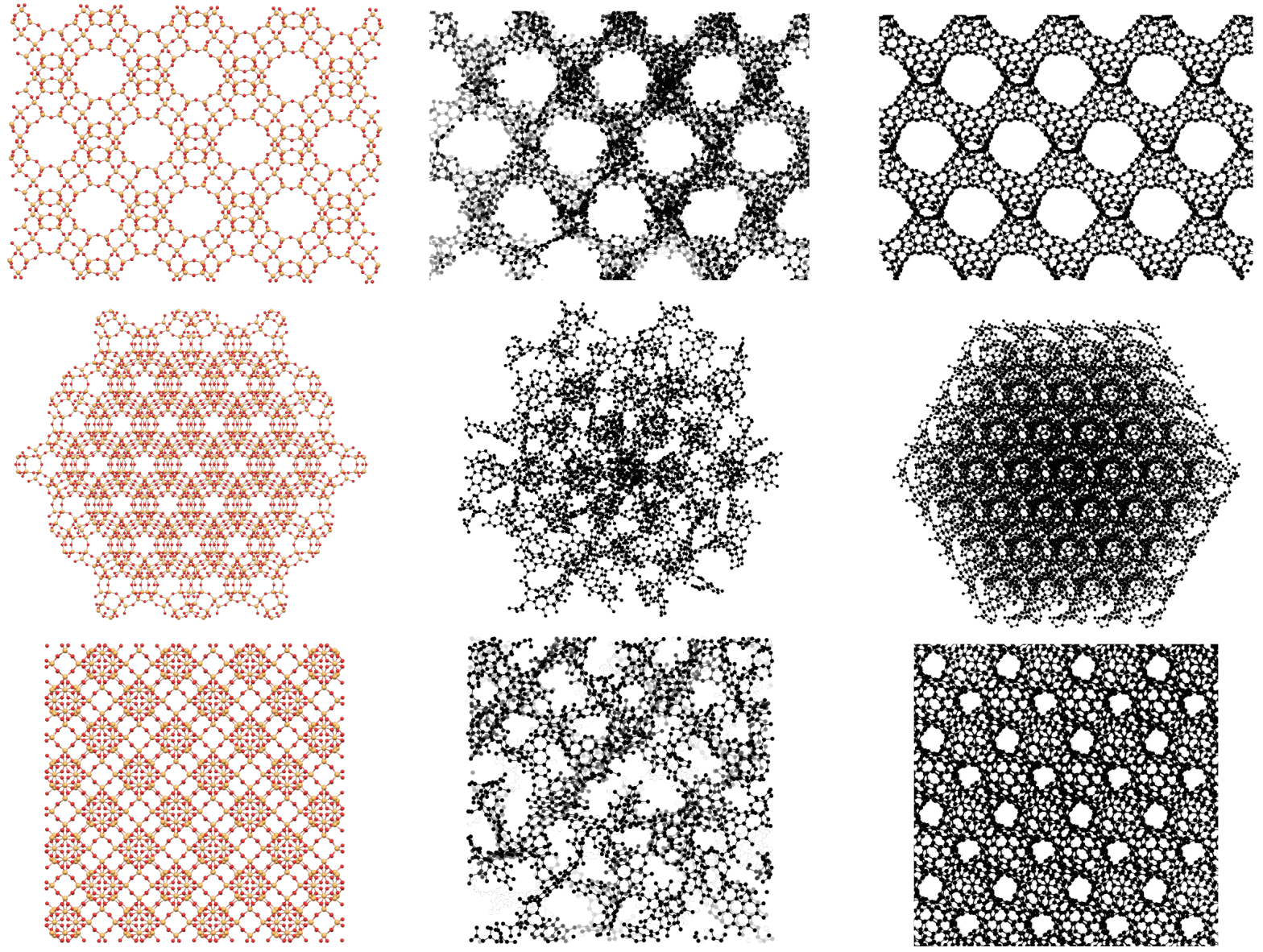

Figure 3. A comparison of a FAU template, Tanaka Model IV, and Braun Model R, viewed from the FAU $\langle 110\rangle$, $\langle 111\rangle$, and $\langle 100\rangle$ directions (top to bottom).

\section{Further Reading}

This work is a summary of review article "Atomistic Structures of Zeolite-Templated Carbon", which can be found at https://doi.org/10.1021/acs.chemmater.0c00535. 


\section{References}

1. Kroto, H. W.; Heath, J. R.; O'Brien, S. C.; Curl, R. F.; Smalley, R. E., C60: Buckminsterfullerene. Nature 1985, 318 (6042), 162-163.

2. Hirsch, A., The era of carbon allotropes. Nat. Mater. 2010, $9(11), 868-871$.

3. $\quad$ Iijima, S., Helical microtubules of graphitic carbon. Nature 1991, 354 (6348), 56-58.

4. $\quad$ Novoselov, K. S.; Geim, A. K.; Morozov, S. V.; Jiang, D.; Zhang, Y.; Dubonos, S. V.; Grigorieva, I. V.; Firsov, A. A., Electric field effect in atomically thin carbon films. Science 2004, 306 (5696), 666-669.

5. $\quad$ Jones, D., Ghostly graphite. Nature 1991, 351 (6327), 526-526.

6. $\quad$ Mackay, A. L.; Terrones, H., Diamond from graphite. Nature 1991, 352 (6338), 762.

7. Lenosky, T.; Gonze, X.; Teter, M.; Elser, V., Energetics of negatively curved graphitic carbon. Nature 1992, 355 (6358), 333-335.

8. Kyotani, T.; Nagai, T.; Tomita, A., Preparation of new carbon from zeolite as template. Proceedings of Carbon 1992, 92, 437-478.

9. $\quad$ Braun, E.; Lee, Y.; Moosavi, S. M.; Barthel, S.; Mercado, R.; Baburin, I. A.; Proserpio, D. M.; Smit, B., Generating carbon schwarzites via zeolite-templating. P. Natl. Acad. Sci. U.S.A. 2018, 115 (35), E8116.

10. Baerlocher, C.; McCusker, L. B. Database of Zeolite Structures. http://www.iza-structure.org/databases/.

11. Nueangnoraj, K.; Nishihara, H.; Imai, K.; Itoi, H.; Ishii, T.; Kiguchi, M.; Sato, Y.; Terauchi, M.; Kyotani, T., Formation of crosslinked-fullerene-like framework as negative replica of zeolite Y. Carbon 2013, 62, 455-464.

12. Boonyoung, P.; Kasukabe, T.; Hoshikawa, Y.; Berenguer-Murcia, Á.; Cazorla-Amorós, D.; Boekfa, B.;

Nishihara, H.; Kyotani, T.; Nueangnoraj, K., A Simple "Nano-Templating” Method Using Zeolite Y Toward the Formation of Carbon Schwarzites. Front. Mater. 2019, 6, 104.

13. Roussel, T.; Jagiello, J.; Pellenq, R. J. M.; Thommes, M.; Bichara, C., Testing the feasibility of using the density functional theory route for pore size distribution calculations of ordered microporous carbons. Mol. Simulat. 2006, 32 (7), 551-555.

14. Nishihara, H.; Yang, Q.-H.; Hou, P.-X.; Unno, M.; Yamauchi, S.; Saito, R.; Paredes, J. I.; Martínez-Alonso, A.; Tascón, J. M. D.; Sato, Y.; Terauchi, M.; Kyotani, T., A possible buckybowl-like structure of zeolite templated carbon. Carbon 2009, 47(5), 1220-1230.

15. Kim, K.; Lee, T.; Kwon, Y.; Seo, Y.; Song, J.; Park, J. K.; Lee, H.; Park, J. Y.; Ihee, H.; Cho, S. J.; Ryoo, R., Lanthanum-catalysed synthesis of microporous 3D graphene-like carbons in a zeolite template. Nature 2016, 535, 131.

16. Gabe, A.; Mostazo-López, M. J.; Salinas-Torres, D.; Morallón, E.; Cazorla-Amorós, D., Synthesis of conducting polymer/carbon material composites and their application in electrical energy storage. In Hybrid Polymer Composite Materials, Elsevier: 2017; pp 173-209.

17. Stadie, N. P.; Vajo, J. J.; Cumberland, R. W.; Wilson, A. A.; Ahn, C. C.; Fultz, B., Zeolite-Templated Carbon Materials for High-Pressure Hydrogen Storage. Langmuir 2012, 28 (26), 10057-10063.

18. Nishihara, H.; Fujimoto, H.; Itoi, H.; Nomura, K.; Tanaka, H.; Miyahara, M. T.; Bonnaud, P. A.; Miura, R.; Suzuki, A.; Miyamoto, N.; Hatakeyama, N.; Miyamoto, A.; Ikeda, K.; Otomo, T.; Kyotani, T., Graphene-based ordered framework with a diverse range of carbon polygons formed in zeolite nanochannels. Carbon 2018, 129, 854-862.

19. Nomura, K.; Nishihara, H.; Yamamoto, M.; Gabe, A.; Ito, M.; Uchimura, M.; Nishina, Y.; Tanaka, H.; Miyahara, M. T.; Kyotani, T., Force-driven reversible liquid-gas phase transition mediated by elastic nanosponges. Nat. Commun. 2019, $10(1), 1-10$.

20. Tanaka, H.; Seto, T.; Nishihara, H.; Kyotani, T.; Miyahara, M. T., Synthesis of zeolite-templated carbons for methane storage: A molecular simulation study. TANSO 2018, 2018(285), 197-203.

21. Sanders, R. Long-sought carbon structure joins graphene, fullerene family. http://news.berkeley.edu/2018/08/13/long-sought-carbon-structure-joins-graphene-fullerene-family/. 22. Nicolaea, S. A.; Wangb, X.; Hedinb, N.; Titiricia, M.-M. Refined biocarbons for gas adsorption and separation. GreenCarbon European Training Network: 2019; p 83.

23. Martin, J. W.; de Tomas, C.; Suarez-Martinez, I.; Kraft, M.; Marks, N. A., Topology of disordered 3D graphene networks. Phys. Rev. Lett. 2019, 123 (11), 116105.

24. Cui, J.; Zhang, K.; Zhang, X.; Lee, Y., A computational study to design zeolite-templated carbon materials with high performance for $\mathrm{CO}_{2} / \mathrm{N}_{2}$ separation. Micropor. Mesopor. Mat. 2019, 109947. 